\title{
Confessions religieuses et processus électoral de 2011: une réflexion sur la manipulation des symboles religieux en République Démocratique du Congo
}

\author{
Joseph Cihunda Hengelela ${ }^{1}$
}

\section{Résumé}

Cet article fait une évaluation critique de l'intervention des Chefs des Confessions religieuses dans le processus électoral de 2011 en République Démocratique du Congo (RDC). Il met en évidence les engagements pris par ces leaders sociaux pour contribuer à un processus électoral transparent, libre et crédible et le non-respect de ces engagements par certains de ces leaders ayant été acquis à la cause du pouvoir en place. L'article plaide pour la dissolution de cette structure de concertation entre les Chefs religieux congolais, car elle n'a pas accompli ses objectifs et appelle chaque communauté religieuse à prendre ses responsabilités devant Dieu, la Nation congolaise et ses fidèles en toute indépendance.

\begin{abstract}
This article is a critical assessment of the role played by the Heads of religious denominations in the 2011 electoral process in the Democratic Republic of Congo (DRC). It highlights the commitments made by these social leaders to contribute to a transparent electoral process, free and credible and non-compliance with these commitments by some of these leaders owing to their allegiance to the political power. The article calls for the dissolution of this dialogue platform between the Congolese religious leaders since it failed to meet their core objectives, and calls each religious community take on its own responsibilities before God, the nation and also before its congregation in complete independence.
\end{abstract}

\section{Introduction}

Les Eglises et les Communautés de foi suivantes s'étaient réunies pour prendre part active au processus électoral de 2011 en la République Démocratique du Congo (RDC). Il s'agit de l'Eglise Catholique, de l'Eglise du Christ au Congo (ECC), de l'Eglise Orthodoxe, de l'Eglise Kimbanguiste, de la Communauté Islamique, de l'Eglise du Réveil du Congo (ERC), de l'Union des Eglises Indépendantes du Congo et de l'Armée du Salut. ${ }^{2}$ Sous une

1 Assistant à la Faculté de Droit de l'Université de Kinshasa, Avocat au Barreau de Kinshasa/Matete et membre du CODESRIA. Chercheur participant au Rule of Law Program de la Fondation Konrad Adenauer. Email : josephcihunda@gmail.com,jcihunda@yahoo.fr

2 Cfr Pour des élections apaisées en RD Congo. Appel des chefs des confessions religieuses, Kinshasa, le 9 août 2011. 
organisation dénommée "Confessions religieuses », ces Eglises et communautés de foi n'étaient pas à leur première occurrence. En 2006, les Confessions religieuses s'étaient également mobilisées pour les élections tant en amont qu'en aval.

Fortes et fières du "succès » de leur première expérience, les Confessions religieuses avaient pris le même engagement pour le processus électoral de 2011. A l'issue de ce processus, elles s'en étaient sorties désunies au détriment de l'unité affichée au départ. Cet état des choses avait réduit sensiblement l'autorité morale des chefs des Eglises et Communautés de foi et ne pouvait pas influer positivement sur le règlement de la crise post-électorale et partant, sur le processus de la consolidation de la démocratie et l'instauration d'un Etat de droit en RDC.

Ainsi cet essai se construit autour de la question de connaître la cause de cette désunion en vue d'en tirer des leçons susceptibles de rendre efficaces les interventions des Confessions religieuses dans le champ politique. Au titre d'hypothèse, je soutiens que la manipulation des symboles religieux par les dirigeants politiques constitue la cause primordiale des contradictions entre les chefs des Confessions religieuses au cours du processus électoral de 2011. Avant de démontrer cette hypothèse, il convient de mettre en évidence le cadre théorique de cette étude et les engagements pris par les Confessions religieuses congolaises à l'égard du dernier processus électoral en RDC.

\section{Cadre théorique de l'étude des interventions des Confessions religieuses dans le processus électoral}

La recherche sur la contribution des Confessions religieuses au processus électoral de 2011 en RDC exige que l'on détermine le cadre d'analyse suivant lequel ce thème sera abordé. Prise dans la perspective juridique, plus particulièrement du droit constitutionnel, les Confessions religieuses qui entrent en commerce avec les pouvoirs publics sont considérées en doctrine comme des groupes de pression. Ainsi que l'écrit Alphonse Daniel Ntumba Luaba $L u m u$, un groupe de pression n'a pas pour ambition de conquérir le pouvoir politique et l'exercer à l'instar des partis politiques. Un groupe de pression, quant à lui, agit sur le fonctionnement des pouvoirs publics pour les influencer dans le sens qui sert ses intérêts. Les actions caractéristiques d'un groupe de pression peuvent revêtir plusieurs formes dont l'information, la consultation, la concertation, la participation et la menace. ${ }^{3}$

Les interventions des Eglises et communautés de foi dans le champ politique sont rendues possibles grâce au principe de la laïcité de l'Etat. Si la laïcité est comprise comme la séparation de l'ordre temporel de l'ordre divin, elle n'exclut pas cependant des interactions entre les deux ordres. La question de la séparation entre l'Eglise et de l'Etat, qui est à

3 Ntumba Luaba Lumu, Droit constitutionnel général, Kinshasa, Editions Universitaires Africaines (EUA), 2007, p. 277. Sur les fonctions des groupes de pression (fonction d'articulation des intérêts, la fonction manifeste de revendication et la fonction latente d'intégration). Voir aussi Mpongo-Bokako Bautolinga E., Institutions politiques et Droit constitutionnel, Tome 1: Théorie générale des institutions politiques de l'Etat, Kinshasa, EUA, 2001, pp. 206-215. 
l'origine de la modernité ${ }^{4}$, n'est pas comprise de la même façon par les acteurs concernés. Cette différence de perception des limites du rôle de l'Etat et des Confessions religieuses est la source de malentendu qui aboutit au conflit ouvert ou latent entre l'Etat et ses partenaires religieux.

En RDC, 1'Etat est laï. ${ }^{5}$ Cette laïcité se traduit en premier lieu par l'absence d'une religion d'Etat. ${ }^{6}$ En d'autres termes, les organisations religieuses sont considérées comme des associations des personnes privées. L'Etat congolais leur attribue le statut des Associations sans but lucratif (ASBL). En effet, le statut des communautés religieuses délimite en luimême l'étendue de la séparation des affaires de l'Etat de celles des Eglises. Il reste dès lors à circonscrire les rapports entre l'Etat, dépositaire du pouvoir temporel et les Confessions religieuses chargées de l'ordre divin sur la terre. La Constitution du 18 février 2006 telle que révisée par la Loi constitutionnelle du 20 janvier 2011 définit clairement la nature des relations entre l'Etat et les Eglises. L'alinéa 2 de l'article 37 stipule:

Les pouvoirs publics collaborent avec les associations qui contribuent au développement social, économique, intellectuel, moral et spirituel des populations et à l'éducation des citoyennes et des citoyens. Cette collaboration peut revêtir la forme d'une subvention.

Les interventions des Confessions religieuses, plus particulièrement de l'Eglise catholique, sur pied de cette disposition de l'alinéa 2 de l'article 37 de la Constitution peut être à l'origine du malentendu entre certains gouvernants et des leaders religieux dans le contexte de l'implication exponentielle des organisations religieuses dans la gouvernance publique en RDC. La nature du problème peut être qualifiée d'incompréhension née à partir du moment où le constituant a délimité le champ de la collaboration avec les associations; champ qui exclut la dimension politique.

En s'attachant uniquement à l'interprétation littérale ou grammaticale de l'alinéa 2 de l'article 37 de la Constitution, la collaboration des pouvoirs publics et des Eglises se limite aux secteurs social, économique, intellectuel, moral et spirituel. Le constituant du 18 février 2006 n'a pas tenu compte du lien intrinsèque entre le domaine politique et les autres domaines de la vie qui exigent que l'on s'occupe primordialement de la politique en vue d'atteindre les résultats voulus dans les autres domaines.

Du côté de l'Eglise catholique, la nature des relations avec les pouvoirs publics n'est pas conçue et entendue au sens grammatical de l'alinéa 2 de l'article 37. Dans la suite du Pape Benoît XVI, les évêques du Congo réaffirmaient cette position qu'ils ont toujours défendue:

4 Lire utilement Amin, S., Modernité et interprétations religieuses, Afrique et Développement, Vol. XXI, n 1,2004 , pp. 7-53.

5 Art. $1^{\text {er }}$ de la Constitution du 18 février 2006, JORDC, $47^{\text {tème }}$ année, Numéro spécial du 18 février 2006.

6 Art. 46 al. 1 de la Loi n ${ }^{\circ}$ 004-2001 portant dispositions générales applicables aux associations sans but lucratif et aux établissements d'utilité publique, JORDC, Numéro Spécial du 15 août 2001. 
...l'Eglise ne peut ni ne doit se mettre à la place de l'Etat, mais elle ne peut et ne doit non plus rester à l'écart dans la lutte pour la justice... Dans son rôle prophétique, chaque fois que le peuple crie vers elle : Veilleurs où en est la nuit..., l'Eglise désire être prête à rendre raison de l'espérance qu'elle porte en elle...car une aube nouvelle pointe à l'horizon... A cause du Christ et par fidélité à sa leçon de vie, notre Eglise se sent poussée à être présente là où l'humanité connait la souffrance et à se faire l'écho du cri silencieux des innocents persécutés, ou des peuples dont les gouvernements hypothèquent le présent et l'avenir au nom d'intérêts personnels. ${ }^{7}$

L'Eglise catholique affirme sa volonté d'être présente partout où l'homme est en souffrance. Dans cette optique, aucun domaine ne va lui échapper, la politique y comprise. En ce qui concerne justement la politique, l'Eglise catholique n'entend pas prendre la place du gouvernement mais elle n'entend pas non plus restée à l'écart de la politique. L'Eglise catholique voudrait restée dans la politique. La question pertinente à se poser est celle de savoir de quel type de politique que l'Eglise voudrait faire. La Conférence Episcopale Nationale du Congo (CENCO) a apparemment répondu à cette préoccupation lorsque les évêques déclaraient ce qui suit : «Nous ne nous lasserons pas de dénoncer tout ce qui met en péril l'édification d'un Etat démocratique $»^{8}$ en RDC.

L'intérêt que présente cette étude sur l'action des Confessions religieuses en rapport avec le processus électoral de 2011 est justement celui de mettre en évidence le rôle des acteurs dans l'émergence de la démocratie et de l'Etat de droit en RDC. En effet, la démocratie et l'Etat de droit ne devaient pas être imaginés comme des institutions qui se décrètent ou qui tombent du ciel par la grâce du bon Dieu ou encore qui sont importées de l'Occident ou de l'Orient pour être placardées dans un pays qui en a besoin. ${ }^{9}$

L'on a aujourd'hui suffisamment les moyens de comprendre que l'Etat démocratique et l'Etat de droit tant recherchés par le peuple congolais se construisent par l'effort de tout citoyen à se conformer aux exigences que cet Etat impose. Il ne suffit pas d'avoir des institutions dites démocratiques pour se dire qu'on est en démocratie ou déclarer que le pays, en l'occurrence la RDC, est un Etat de droit, comme l'a fait le constituant du 18 février 2006, pour se croire dans un Etat de droit aujourd'hui. Les nombreux faits et actes vécus de 2006 à 2015 suffisent pour démontrer l'écart entre les prescriptions constitutionnelles et la réalité vécue sur le terrain.

La démocratie et l'Etat de droit en RDC deviendront une réalité avec l'implication soutenue et sans complaisance de tous les acteurs. Les Confessions religieuses sont l'un de ces acteurs sociaux dont le rôle demeure prépondérant pour empêcher ceux qui veulent

7 Benoît XVI, Exhortation apostolique post-synodale Africae munus, par. 30.

$8 C E N C O$, Le peuple congolais a faim et soif de justice et de paix. Le courage de la vérité (cf. 2 Co 7 , 14). Message de l'Assemblée plénière extraordinaire de la CENCO aux fidèles catholiques et à l'ensemble du peuple congolais, Kinshasa, Editions du Secrétariat général de la CENCO, 2012, par. 8.

9 Dans le même sens, Cihunda Hengelela J., Acteurs de la bonne gouvernance en RD Congo postélectorale, Congo-Afrique (Mars 2008) n 423, pp. 203-216. 
s'écarter du processus démocratique dans le but de replonger le pays dans une nouvelle dictature. ${ }^{10}$ Quel est le degré de prise de conscience de cette responsabilité par les Chefs religieux ? Si ce degré est élevé, est-il traduit en actes concrets?

\section{Engagements des Confessions religieuses à l'égard du processus électoral de 2011}

Les Chefs des Confessions religieuses congolaises se sont engagés dans le processus électoral de 2011 comme ils l'avaient fait lors des élections présidentielle et legislatives de 2006. Ils avaient tenu à expliquer que leur démarche était une initiative de paix, de fraternité, de service et d'amour en faveur de la RDC et de son peuple. Selon eux, Dieu leur avait confié la mission de prêcher la bonne nouvelle de la paix à temps et à contretemps, en tout lieu et en tout temps, quelles que soient les circonstances, à tous les hommes, quels que soient leur rang et leur qualité. S'ils ne pouvaient pas prêcher cette nouvelle, ils failliraient à leur vocation de messagers de la paix. ${ }^{11}$

Sûrs de leur autorité morale sur l'ensemble de leurs fidèles, les Chefs de Confessions religieuses entendaient « ...jouer, pour l'avenir de notre pays, le 'rôle d'interpellateur objectif des consciences, sans complaisance ni parti-pris'. Pour les Chefs religieux, leur silence serait considéré comme leur complicité. Ce qui est, à leurs yeux, une catastrophe pour le pays. ${ }^{12}$

Se situant dans leur rôle d'interpellateur objectif des consciences, les Chefs religieux se disaient ne prendre position pour aucun parti politique. Ils ne pouvaient pas non plus donner des consignes de vote en faveur ou en défaveur d'un candidat. Cet engagement pouvait changer « ...en cas d'atteinte grave à la morale, à la dignité de la personne humaine » et à leurs convictions religieuses $»{ }^{13}$ Pourtant, ce que les Chefs religieux craignaient plus au 9 août 2011 se produisait déjà depuis le mois de janvier 2011 à la suite de la révision de la Constitution du 18 février 2006. Cette période, allant de janvier à décembre 2011, a été caractérisée par des atteintes graves à la morale, à la dignité humaine et aux convictions religieuses.

En ce qui concerne les dérapages des processus électoraux ayant débouché sur les violences, les Chefs religieux faisaient observer que les cas semblables et malheureux de certains pays d'Afrique étaient déjà vécus en ce mois d'août 2011 en RDC. D'où l'urgence

10 Bakandeja wa Mpungu G., Avant-propos, in Bakandeja wa Mpungu G., Mbata Betukumesu Mangu A. et Kienge-Kienge Intudi $R$. (dir.), Participation et responsabilité des acteurs dans le contexte d'émergence démocratique en République Démocratique du Congo. Actes des journées scientifiques de la Faculté de Droit de l’Université de Kinshasa 18-19 juin 2007, Kinshasa, Presses Universitaires de Kinshasa (PUK), 2007, p. 11.

$11 \S 2$, Pour des élections apaisées en RD Congo. Appel des chefs des confessions religieuses,...op. cit.

$12 \S 3$, Pour des élections apaisées en RD Congo. Appel des chefs des confessions religieuses,...op. cit.

$13 \S 4$, Pour des élections apaisées en RD Congo. Appel des chefs des confessions religieuses,...op. cit. 
d'un engagement de la part des interpellateurs de conscience. Ainsi cet engagement se fit par l'appel des Chefs de Confessions religieuses aux acteurs intéressés par ce processus electoral. Cet appel des Chefs de Confessions religieuses contenait des recommandations qu'il convient d'examiner.

\subsection{Recommandations aux partis politiques}

Les partis politiques ont été les plus visés par les Chefs de Confessions religieuses. Les recommandations à leur égard peuvent être groupées en quatre catégories. En effet, les Chefs religieux recommandaient aux partis politiques d'être acquis aux vertus démocratiques. Pour eux, être acquis aux vertus démocratiques signifie:

- respecter la Constitution et les lois de la République auxquelles tout le monde doit être soumis;

- accepter le pluralisme politique;

- avoir confiance dans les institutions du pays et se soumettre au Code de bonne conduite;

- refuser de recourir aux pratiques de la corruption;

- rejeter l'impunité;

- régler les conflits électoraux par voie judiciaire;

- avoir la retenue et la maîtrise de soi; et

- s'interdire de jouer avec le feu de la division et s'abstenir d'attiser les brasiers de la haine.

La deuxième série des recommandations aux partis politiques est liée au profil des candidats et au projet de société à proposer aux électeurs congolais. Pour les Chefs de Confessions religieuses, les candidats aux élections devaient répondre au profil et devaient s'engager sur la base d'un projet de société. Pour eux, répondre au profil signifie:

- avoir un sens élevé de responsabilité et de patriotisme;

- avoir une sagesse avérée, une maîtrise de soi, une retenue éprouvée (les candidats doivent être des hommes et des femmes...qui arrivent au pouvoir par des élections justes, c'est-à-dire transparentes et crédibles);

- savoir respecter la Constitution et les textes en vigueur et viser le bien commun;

- se faire voter sur la base d'un projet de société en lieu et place des cadeaux aboutissant à l'achat des consciences;

- répondre au profil de compétence, de loyauté, d'intégrité et de moralité;

- laisser le peuple voter en âme et conscience, en toute responsabilité, à la suite d'une comparaison et d'une évaluation des candidats sur la base de leurs programmes; et

- respecter la volonté du peuple.

La troisième catégorie des recommandations est relative au respect du verdict des urnes.

Les Chefs religieux avaient recommandé aux partis politiques et aux candidats de:

- exprimer l'engagement personnel et individualiser de respecter le verdict des urnes;

- multiplier les faits et gestes pour inspirer confiance et lutter contre les soupçons qui caractérisent les élections; 
- éviter les contestations tendancieuses et hasardeuses des suffrages qui relèvent de la mauvaise foi;

- accepter la défaite avec humilité; et

- savourer la victoire sans triomphalisme.

La quatrième série de recommandations est un appel à la crainte de Dieu. Pour les Chefs religieux congolais, les responsables des partis politiques devaient craindre Dieu pour avoir la sagesse qui les aidera à conduire les affaires publiques. Pour eux, la sagesse qui vient de Dieu commande que la direction des affaires du pays soit confiée:

- à des hommes et des femmes compétents;

- aux hommes et aux femmes qui se laissent guider par la parole de Dieu;

- à des personnes de bonne volonté, des hommes et des femmes vertueux qui soient attentifs aux valeurs démocratiques et éthiques; et

- à des personnes qui savent se sacrifier pour un idéal noble, celui du service pour le bien commun du peuple congolais.

Je n'ai pas du mal à identifier à quel parti politique les Chefs religieux s'adressaient; dans la mesure où ce message paraissait comme une thérapie contre les maux qui minent le fonctionnement de tous les partis politiques en RDC. Jusqu'au 15 mars 2015, on dénombrait 477 partis politiques enregistrés au Ministère des affaires intérieures. Il est difficile de faire leur classification en fonction de leur idéologique qui par ailleurs est introuvable dans la plupart de ces partis. La preuve est donnée par la constitution des coalitions ou regroupements politiques qui se fait non pas sur la base idéologique mais souvent et surtout en fonction des intérêts personnels de chefs ou initiateurs des partis politiques.

On peut cependant mettre en exergue les partis politiques à resonance religieuse réportiés dans le tableau suivant.

\section{Tableau des partis politiques d'inspiration religieuse}

\begin{tabular}{|l|l|l|}
\hline$N^{\circ}$ & Dénominations officielles & Sigles \\
\hline 1 & Alliance des Bâtisseurs Chrétiens & ABC \\
\hline 2 & Alliance Congolaise des Démocrates Chrétiens & ACDC \\
\hline 3 & Alliance des Chrétiens pour la Démocratie et le Développement & ACDD \\
\hline 4 & Alliance des Chrétiens pour le Renouveau et le Progrès & ACRP \\
\hline 5 & Alliance des Démocrates Chrétiens congolais & ADC \\
\hline 6 & Alliance Chrétienne pour le Progrès & AICP \\
\hline 7 & Alliance des Nationalistes Croyants Congolais & ANCC \\
\hline 8 & $\begin{array}{l}\text { Alliance du Peuple pour le Plan de Dieu en République Démocratique du } \\
\text { Congo }\end{array}$ & APPD/RDC \\
\hline 9 & Convention des Chrétiens et Croyants pour le Changement & CCCC \\
\hline 10 & Convention Chrétienne pour la Démocratie & CCD \\
\hline 11 & Chrétiens Démocrates & CD \\
\hline
\end{tabular}




\begin{tabular}{|c|c|c|}
\hline $\mathrm{N}^{\circ}$ & Dénominations officielles & Sigles \\
\hline 12 & Convention des Démocrates Chrétiens & $\mathrm{CDC}$ \\
\hline 13 & Congrès Islamique pour le Développement & CID \\
\hline 14 & Convergence Chrétienne & $\mathrm{COC}$ \\
\hline 15 & $\begin{array}{l}\text { Démocratie Chrétienne Fédéraliste-Convention des Fédéralistes pour la Dé- } \\
\text { mocratie Chrétienne }\end{array}$ & COFEDEC \\
\hline 16 & Convention Nationale pour la Démocratie Chrétienne & CONADEC \\
\hline 17 & Conseil Patriotique et Progressiste Chrétien & CPPC \\
\hline 18 & Démocratie Chrétienne & DC \\
\hline 19 & Dynamique Chrétienne pour un Congo Nouveau & DCCN \\
\hline 20 & Démocratie Chrétienne Fédéraliste/Nyamwisi & DCF \\
\hline 21 & Front des Indépendants pour la Démocratie Chrétienne & FIDEC \\
\hline 22 & Forum des Chrétiens pour la Reconstruction Nationale & FORENAC \\
\hline 23 & Ligue des Démocrates Chrétiens & LDC \\
\hline 24 & Ligue des Patriotes et Démocrates Socio-Chrétiens & LPDSC \\
\hline 25 & Mouvement Chrétien Congolais & MCC \\
\hline 26 & Mouvement Chrétien pour le Développement Intégral & MCDI \\
\hline 27 & Mouvement Chrétien pour la Solidarité et la Démocratie & MCSD \\
\hline 28 & Mouvement Démocrate Chrétien & MDC \\
\hline 29 & Parti pour l'Amour du Prochain et de la Patrie & PAPP \\
\hline 30 & $\begin{array}{l}\text { Parti de la Protection d'Allah et son Prophète Mohamed Roi Souverain des } \\
\text { Hommes }\end{array}$ & PAPRAHORODE \\
\hline 31 & Parti Chrétien Authentique & PCA \\
\hline 32 & Parti Chrétien Démocrate pour le Développement Intégral du Congolais & PCDI \\
\hline 33 & Parti Chrétien des Démocrates Libéraux & PCDL \\
\hline 34 & Parti Chrétien pour la Solidarité Africaine & PCSA \\
\hline 35 & Parti Démocrate Chrétien & PDC \\
\hline 36 & Parti Démocrate et Social Chrétien & PDSC \\
\hline 37 & Parti Fédéral Chrétien & $\mathrm{PFC}$ \\
\hline 38 & Parti Libéral Démocrate Chrétien & PLDC \\
\hline 39 & Reveil Chrétien & $\mathrm{RC}$ \\
\hline 40 & Rassemblement des Chrétiens Démocrates pour le Progrès & RCDP \\
\hline 41 & Rassemblement des Chrétiens pour le Congo & RCPC \\
\hline 42 & Rassemblement des Chrétiens Républicains & RCR \\
\hline 43 & Rassemblement des Démocrates Chrétiens & $\mathrm{RDC}$ \\
\hline 44 & Rassemblement des Missionnaires Démocrates pour la Justice & RMDJ \\
\hline 45 & Union Chrétienne pour la Libération des Opprimés & UCLO \\
\hline 46 & Union des Chrétiens Républicains & UCR \\
\hline 47 & Union Chrétienne pour le Renouveau et la Justice & UCRJ \\
\hline 48 & Union des Démocrates Chrétiens & UDC \\
\hline
\end{tabular}




\begin{tabular}{|l|l|l|}
\hline $\mathrm{N}^{\circ}$ & Dénominations officielles & Sigles \\
\hline 49 & Union des Démocrates Chrétiens Fédéralistes & UDECF \\
\hline 50 & Union des Démocrates et Sociaux Chrétiens & UDSC \\
\hline 51 & Union des Libéraux Démocrates Chrétiens & ULDC \\
\hline 52 & Union Nationale des Démocrates Chrétiens & UNADEC \\
\hline 53 & Union des Patriotes Démocrates Chrétiens & UPDC \\
\hline 54 & Union des Républicains Chrétiens & URC \\
\hline
\end{tabular}

Source: tableau fait à partir de la liste des partis politiques transmise à la Commission électorale nationale indépendante par le Ministre de l'Intérieur.

Faire une recommandation aux partis politiques congolais d'avoir la crainte de Dieu peut paraître comme un vœu pieux. Sur les 477 partis régulièrement inscrits, 54 seulement contiennent les termes « Dieu », « chrétien », " Allah », « Islamique », " Mohamed » et « Croyant » dans leurs dénominations. Ce qui représente $11,3 \%$ du paysage partisan congolais. Il reste à savoir ce qui est intrinsèquement religieux en ces partis. Eu égard à leur fonctionnement, la plupart des partis politiques congolais ont leur « dieu » qui est soit le pouvoir, soit l'argent, soit encore les deux à la fois.

Pour les partis politiques de la coalition au pouvoir, le vrai Dieu n'est bon que lorsqu'il sert à protéger leur pouvoir. Au cas contraire, il n'est qu'un refuge pour les pauvres qu'ils condamnent à demeurer dans cet état. Massacrer les fidèles qui dérangent le régime, assassiner les religieux qui entêtent ces fidèles ou profaner les lieux des cultes en y envoyant les policiers y jeter les gaz lacrymogène ; tant que tout concourt à consolider le pouvoir, Dieu n'a qu'à faire de lui-même. Tant pis pour ceux qui croient en lui. Dans cet environnement, il est illusoire de penser qu'il y a en RDC un parti politique qui allait sacrifier ses intérêts pour la crainte de Dieu en observant ainsi la recommendation des Chefs des Confessions religieuses. Tant que le pouvoir de sanctionner positivement ou négativement les dirigeants ne sera pas restitué au peuple par le respect de la vérité des urnes, les partis politiques continueront toujours à se jouer du peuple congolais. La colère de Dieu ne semble qu'une promesse lointaine pour eux.

Le peuple congolais avait été la deuxième cible des recommandations des Chefs religieux qui prétendaient s'engager dans le processus électoral pour son bien matériel alors que le bien spirituel est assuré par eux-mêmes.

\subsection{Recommandations au peuple congolais}

Les Chefs religieux recommandaient au peuple congolais « d'avoir jalousement à l'esprit son statut de 'souverain primaire' ». Pour eux, le statut de souverain primaire signifie:

- que chaque citoyen, par le droit de vote, détient une part de la souveraineté nationale;

- que l'exercice de ce droit appelle une vigilance avant, pendant et après les élections; 
- que chaque citoyen jouit de la liberté de conscience et a le fair-play, il cultive les vertus démocratiques; et

- qu'il vote avec responsabilité.

Ces recommandations au peuple congolais n'appellent même pas des commentaires à cause du fait que ce peuple a suffisamment démontré qu'il était mûr. Il a été toujours trahi par ses dirigeants. Le peuple congolais a réellement conscience de son statut de souverain primaire. Il lui reste de trouver les moyens de se faire respecter en tant que tel. L'effort supplémentaire qu'il faut peut-être demander à tous les dirigeants congolais, y compris les Chefs religieux, de prendre au sérieux ce peuple et de le respecter en tant que souverain primaire. C'est qui n'a pas toujours été le cas tant en 2006 qu'en 2011.

\subsection{Recommandations à la Commission Electorale Nationale Indépendante (CENI)}

Les Chefs des Confessions religieuses avaient recommandé à la CENI de:

- poser des faits et gestes qui rassemblent;

- mettre en place « des mécanismes adaptés à une résolution prompte, juste et équitable des incidents électoraux »; et

- de faire fi de leurs appartenances politiques pour retrouver le difficile et délicat rôle de neutralité en veillant à la transparence.

Pour les Chefs religieux, seule la transparence était à même d'apaiser le climat politique et inspirer confiance. Elle était une condition sine qua non de l'acceptation des résultats sortis des urnes. L'insistance sur la transparence peut étonner au regard du mutisme que les Chefs religieux avaient gardé lorsque les partis politiques de l'Opposition réclamaient l'audit du fichier électoral. Devant l'exigence des partis de l'Opposition qui demandaient que le fichier électoral soit audité avec la participation de leurs représentants, la CENI qui semblait acquiescer à cette demande, s'était ravisée avec le soutien du Gouvernement pour rejeter carrément cette demande. C'est à partir du fichier électoral que la fraude aurait été préparée. La position des Chefs religieux dans ce débat n'a pas été connue.

\subsection{Recommandations aux médias}

Les Chefs religieux demandaient aux médias de:

- donner une information correcte pour jouer un rôle important dans la participation citoyenne en démocratie;

- faciliter l'égal accès des candidats aux médias publics avec un grand sens de responsabilité;

- donner à la société une information fondée sur la vérité, la liberté, la justice et la solidarité; et

- de se conformer au Code de déontologie de leur métier, à « ne pas déformer, dénaturer ou fausser, par leur formulation, par insistance, grossissement, omission ou manipula- 
tion les opinions d'autrui, les titres ou les commentaires des articles qui doivent être traités avec impartialité et bonne foi.

Cette recommandation peut donner l'impression que les Chefs religieux ne suivent pas les médias de la capitale, soit ils vivent tous à l'intérieur du pays. Ce qui est loin d'être une vérité. Le paysage médiatique congolais est bipolarisé, à l'exception de quelques groupes de presse qui tiennent encore à la noblesse de leur métier. En effet, il existait à l'époque de leur appel pour les élections apaisées, des médias et des journalistes du pouvoir et ceux de l'Opposition. Les médias publics ont été toujours acquis à la cause du tenant du pouvoir présidentiel. La Radiotélévision Nationale Congolaise (RTNC) n'a jamais été une « église au milieu du village ». Une grosse illusion qui ne contente que ses auteurs. Elle est " une cabane » au coin du village.

Les Chefs religieux étaient confiants en insistant sur cette recommandation comme à celles qu'ils avaient faites au Gouvernement de la République oubliant le fait que ce même Gouvernement avait ordonné la fermeture de certains médias appartenant aux Opposants au régime en place.

\subsection{Recommandations au Gouvernement de la République}

Pour les Chefs des Confessions religieuses, le Gouvernement avait un rôle irremplaçable dans l'encadrement du processus électoral, notamment pour:

- allouer des ressources financières et logistiques nécessaires aux différentes opérations électorales; et

- assurer l'impartialité de l'armée, de la police et des services de sécurité, ainsi que des mécanismes indispensables pour garantir l'indépendance de la CENI.

Ils recommandaient au Gouvernement de:

- faire de la réussite des élections un point d'honneur à la hauteur de la vocation et de la grandeur de la RDC;

- veiller à l'exemplarité en ce qui concerne le respect de la Constitution et des lois;

- mettre en place des mécanismes garantissant la transparence sur les sources de financement des campagnes, la sécurité et la protection de la population et des candidats;

- faciliter l'accès équitable aux médias pendant tout le processus électoral; et

- avoir une attitude responsable, avec des discours et des actions qui rassurent pour un déroulement des élections dans la transparence, la vérité et la paix.

Une fois de plus, les Chefs religieux congolais donnaient l'impression de s'être enfermés dans un carcan idéologique qui les empêchait de voir et de dire la vérité. Avaient-ils oublié que les militaires, les policiers et les agents de sécurité n'étaient pas neutres et qu'ils étaient placés au service individualisé des tenants du pouvoir? La vocation de la grandeur de la RDC ne pouvait pas se mesurer à l'organisation d'une élection. Il paraissait vain d'exiger des dirigeants moins enclins à l'esprit de grandeur de réaliser les choses qui dépassent leurs limites et leur vision politique. 
Après cinq ans de gouvernance, les animateurs des institutions politiques avaient fait preuve d'incapacité notoire à se départir des antivaleurs. L'injustice, l'impunité, le népotisme, le régionalisme, le tribalisme, le clanisme, la corruption, le clientélisme et l'incompétence avaient refait surface accentuant la ruine de tous les efforts consentis pour le relèvement du pays après quinze ans de guerre. Le Président Joseph Kabila était conscient de cet échec. Il n'avait pas intérêt à organiser des élections transparentes qu'il devait perdre. Son grand projet qui semble lui tenir à cœur est de rester indéfiniment au pouvoir. Tous les débats autour de la révision de la Constitution du 18 février 2006 pour lui offrir la possibilité de se présenter pour une troisième fois à l'élection présidentielle rentre dans cette logique de se maintenir au pouvoir. C'est qui incite ses courtisants à concocter des scénarii qui peuvent rendre possible ce projet.

Dans ce contexte, aucune recommandation des Chefs des Confessions religieuses n'avait la chance d'être respectée. Ainsi, les recommandations des Chefs des Confessions religieuses n'étaient pas suivies par leurs destinataires, sauf peut-être le peuple congolais qui s'était non seulement rendu massivement voter sous une pluie battante, mais aussi est resté à maintes endroits, vigilant pour veiller au déroulement du double scrutin.

Contre toute attente, le peuple congolais a été victime des machinations orchestrées et ayant abouti au détournement de sa volonté exprimée dans les urnes. Ce qui nous importe maintenant est de voir quelle a été l'attitude de ces Chefs religieux à l'issue du double scrutin du 28 novembre 2011? Etaient-ils encore unis à l'égard du déferlement de la violence, des fraudes électorales et de toutes les antivaleurs qu'ils ne partagent pas?

\section{Désunion dans la réaction à l'égard des résultats des élections du 28 novembre 2011}

Les résultats des élections présidentielle et législatives du 28 novembre 2011 avaient été jugés non crédibles par la majorité des Congolais, par toutes les Organisations Non Gouvernementales $(\mathrm{ONG})$ nationales et internationales ainsi que par une partie de la Communauté internationale. La Société civile congolaise à laquelle appartiennent également les Confessions religieuses avait adressé une lettre au Secrétaire des Nations Unies au sujet de la crise postélectorale. On peut lire dans cette lettre ce qui suit:

Point n'est besoin de revenir aux graves constats du chaos et à l'ampleur incommensurable de la fraude qui ont caractérisé la préparation, l'organisation, le dépouillement et la proclamation/publication des résultats des élections présidentielle et législatives du 28 novembre 2011 en RDC. Le manque de crédibilité de ces élections a été reconnue par tous les observateurs : l'Union européenne, le Centre Carter, les observateurs nationaux, les observateurs de l'Eglise Catholique, ... ${ }^{14}$

14 Lettre de la Société civile congolaise au Secrétaire général des Nations Unies sur la crise post-électorale en RDC, Kinshasa, le 7 février 2012. 
Cette lettre dans laquelle la Société civile soutenait qu'elle ne se reconnaissait pas dans les résultats publiés par la CENI, était signée également par les observateurs des Confessions religieuses (ROC). Devant ces résultats, l'attitude des Chefs des Confessions religieuses oscilla entre le mutisme et la désunion en face d'une situation ostentatoirement à l'antipode de leurs attentes et aux conséquences fâcheuses pour l'avenir du pays.

\subsection{Eclosion de la désunion entre les Chefs religieux face aux résultats du processus électoral}

Du 28 novembre au 9 décembre 2011, il s'était passé un nombre impressionnant d'événements qui allait emporter la crédibilité de tout le processus électoral. De toutes les huit Confessions religieuses, seule l'Eglise Catholique fit une déclaration le 3 décembre 2011. A cette date précise, les 6.000 observateurs catholiques avaient réussi à transmettre à la CENCO 46\% des réponses sur l'état du déroulement des élections.

Il résultait des conclusions des observateurs catholiques des enseignements tant à la participation qu'aux irrégularités. Au sujet des irrégularités justement, la CENCO s'était réservée d'entrer en détail; mais elle réclamait des sanctions proportionnelles à l'ampleur de la fraude constatée. Au moment où les évêques lançaient leur appel, la tension dans toutes les villes du Congo était perceptible. On avait en esprit les cas malheureux des violences postélectorales au Kenya, ${ }^{15}$ au Zimbabwe ${ }^{16}$ et en Côte d'Ivoire.

Au 9 décembre 2011, les résultats provisoires de l'élection présidentielle étaient proclamés donnant Joseph Kabila Kabange vainqueur avec 48,9\% des suffrages. Ces résultats proclamés par la CENI étaient contestés et avaient donné lieu à des actes de violence. La ville de Kinshasa, la capitale de la RDC, fut assiégée par des chars de la Garde présidentielle pendant plus d'une semaine jusqu'à la prestation du serment par le Président réélu le 20 décembre 2011. Face à ces résultats, la première réaction est celle du Cardinal Laurent Monsengwo Pasinya. Au cours d'un point de presse qu'il avait tenu le 12 décembre 2011, le Cardinal Laurent Monsengwo déclara que les résultats de l'élection présidentielle annoncés le 9 décembre 2011 n'étaient pas conformes à la vérité ni à la justice. Un jour après, Monseigneur Pierre Marini Bodo, représentant légal de l'Eglise du Christ au Congo (ECC), contredisait le Cardinal Monsengwo soutenant que les résultats publiés étaient conformes à la vérité et à la justice.

Quant à l'Eglise catholique, il a fallu attendre le 11 janvier 2012 pour que la CENCO fît une déclaration au sujet des résultats publiés par la CENI. Au cours de l'Assemblée plénière extraordinaire de la $\mathrm{CENCO}$, les membres de cette institution ecclésiale appuyèrent la déclaration du Cardinal Monsengwo à propos de la non-conformité à la vérité

15 Lire Murunga G.R., Les élections générales au Kenya : propagande politique troublante sous couvert de débat intellectuel, Bulletin du CODESRIA, $n^{\circ} 1 \& 2,2009$, pp. 17-25.

16 Dans ce sens, voir Mangezvo P.L., Souveraineté, scrutin et démocratisation : analyse des processus politiques et électoraux au Zimbabwe au cours du début du vingt et unième siècle, Bulletin du CODESRIA, $n^{\circ} 1 \& 2,2009$, pp. 32-41. 
des urnes des résultats de l'élection présidentielle publiés par la CENI. L'on peut tirer du message des évêques congolais ce qui suit:

Aujourd'hui, il ressort du rapport final de la mission d'observation électorale de la CENCO et des témoignages recueillis des divers diocèses et d'autres sources que le processus électoral s'est déroulé, à beaucoup d'endroits, dans un climat chaotique. L'on a noté plusieurs défaillances, des cas de tricheries avérées et vraisemblablement planifiées, de nombreux incidents malheureux entrainant mort d'hommes, des cafouillages, et, à certains endroits, un climat de terreur entretenu et exploité à dessein pour bourrer les urnes. Ce n'est pas tout. Ce qui se passe présentement au niveau de la compilation des résultats des élections législatives est inacceptable. C'est une honte pour notre pays. ${ }^{17}$

Eu égard à ce qui précède, les évêques estimaient que le processus électoral avait été entaché de graves irrégularités qui remettaient en question la crédibilité des résultats publiés par la CENI.

Hormis, ces quatre déclarations dont deux faites par des personnalités responsables au plus haut niveau de leurs communautés religieuses respectives et deux engageant directement l'Eglise Catholique, les autres Confessions religieuses brillèrent par un silence coupable. Ce silence péchait contre l'engagement que les Chefs des Confessions religieuses avaient pris quatre mois plus tôt. D'où la nécessité de réfléchir sur les causes à la base de l'attitude on dirait irresponsable de certains Chefs religieux congolais.

\subsection{Manipulation des symboles religieux par les dirigeants politiques au pouvoir}

Le pouvoir mobilisateur des Confessions religieuses n'a pas encore servi la cause de la démocratie ni de l'Etat de droit au cours cette troisième République en RDC. L'interventionnisme religieux dans la gouvernance publique se heurte à une volonté du statu quo du régime ayant opté pour l'autoritarisme presque vulgaire. Les pouvoirs publics tentent, parfois avec un succès relatif, à soumettre les dignitaires religieux en échange de l'octroi des avantages à la fois matériels et moraux. Dans ce contexte, il parait difficile pour les Chefs des Confessions religieuses de prétendre infléchir les actions des autorités politiques.

Le premier facteur qui entre dans la stratégie de la manipulation des symboles religieux est le déséquilibre des forces entre les Confessions religieuses et le régime au pouvoir. Ce rapport des forces ne changera pas tant que les Confessions religieuses, comme organisation, continueront à ressembler à un panier à crabes où l'on peut trouver des «saints » et des « diables », de « bons grains » et de l' « ivraie ». Toutes les Eglises et Communautés

17 CENCO, Le Peuple congolais a faim et soif de justice et de paix. Le courage de la vérité (cf. 2 Co $7,14)$. Message de l'Assemblée plénière extraordinaire de la CENCO aux fidèles catholiques et à l'ensemble du peuple congolais, Kinshasa, Editions du Secrétariat Général de la CENCO, 2012, p. 5 . 
religieuses sont actuellement infiltrées par les "agents secrets" du régime dans la perspective des élections de 2016. L'Eglise Catholique y comprise.

Pour se pérenniser au pouvoir, la nécessisté de recourir aux symboles religieux se conjugue avec les efforts que déploient les juristes constitutionnels du palais présidentiel. Des prophéties sont distillées annonçant la volonté de Dieu de voir le Président de la République rempiler. Dans la période électorale, alors que les strategies de fraude à l'élection avait été mises en place depuis la revision constitutionnelle qui avait ramené de deux à un seul tour l'élection du Président de la République, de nombreux pasteurs ont demandé à leurs fidèles de voter pour le Président sortant qui était le candidat de Dieu.

A partir de 2012, une série de prophéties vont se rependre au sujet de la volonté de Dieu sur le Président de la République. La première et la plus comique est qu'un enfant serait né quelque part dans le Grand Kivu portant sur son corps le nom du Président de la République. A cette époque, nous sommes au début du mandat. Vers la fin de ce mandat, la prophétie a touché le coeur de la foi "rationnelle", l'Eglise catholique. Au sein des mouvements feministes les plus engagés, en l'occurrence les "Mamans catholiques", Dieu aurait révélé à l'une d'entre elles sa volonté sur la RDC: le Président actuel regnera jusqu'en 2021. C'est ce que le descendant de l'Envoyé special de Jésus-Christ sur la terre (Chef spirituel de l'Eglise Kimbanguiste) aurait confirmé.

Les exemples de manipulation des symboles religieux sont légions. Lorsque les manipulations ne sont pas les faits directs des responsables religieux, ils émanent des services de renseignement rompus à ces pratiques. Recourir aux symboles religieux pour se maintenir au pouvoir n'est pas une nouveauté en RDC. Le feu Président Mobutu fit de même jusqu'à devenir lui-même une divinité que ses courtisans adoraient.

Le deuxième facteur est constitué par le fait que la corruption a atteint beaucoup de Chefs religieux. Par voie directe ou indirecte, les « hommes de Dieu » n'échappent pas au cancer de la corruption qui gangrène la vie publique en RDC. Ils se servent de leur position sociale pour s'enrichir. C'est ce qui peut expliquer des réactions épidermiques et hasardeuses ou le mutisme devant les situations qui appellent la défense de l'intérêt public. La corruption directe consiste en la perception de l'argent et des biens matériels. La bonne et consistance " dîme » est celle payée par les politiciens. Les Eglises se construisent également avec les tôles et les sacs de ciment donnés par les politiciens qui investissent les lieux de culte in tempore suspecto, c'est-à-dire pendant les périodes de campagnes électorales.

En contrepartie, les Chefs religieux bénéficiaires donnent des consignes de vote à travers les prophéties sur l'élection du candidat donateur. Les Chefs religieux se disent avoir reçu un message de « Dieu » recommandant aux fidèles de porter leur choix sur son « oint ». A l'approche de la date du 28 novembre 2011, des sermons pareils ont été entendus dans la plupart des églises. Une telle attitude était une violation de l'un de leurs engagements pris le 9 août 2011.

La corruption indirecte, quant à elle, se traduit par des cadeaux offerts par les dirigeants politiques à l'occasion de sacre des évêques ou d'autres dignitaires religieux. Il en est de même des visites de courtoisie aux Chefs religieux aux temples ou mosquées. L'un des 
lieux « saints » les plus visités par les politiciens est la cité de Nkamba, considérée par les Kimbanguistes comme la Jérusalem africaine. L'année 2011 était très particulière pour l'Eglise Kimbanguiste qui est traditionnellement attachée au pouvoir. C'est une Eglise de tous les régimes et du statu quo. Pour cette Eglise africaine, cette année 2011 coïncidait avec le quatre-vingt-dixième anniversaire (1921-2011) de la condamnation à mort par le Tribunal de première instance de Thysville (Mbanza-Ngungu) de son fondateur, le Prophète Simon Kimbangu. Un jugement de révision avait été obtenu et signifié à Mbanza-Ngungu avec l'appui total du Gouvernement. L'on ne pouvait pas hésiter sur le soutien que cette Eglise devait apporter au candidat du pouvoir à l'élection présidentielle.

La liste des facteurs de la désunion des Confessions religieuses face aux résultats de l'élection présidentielle de 2011 peut être allongée. Ce qui importe est l'incidence du comportement des Chefs religieux sur le processus électoral en RDC. La conséquence immédiate de ce comportement est la réémergence du phénomène d'une « Eglise d'en haut » et d'une « Eglise d'en bas » au sein des Communautés religieuses en RDC. C'est à ce niveau qu'il convient de se poser la question de savoir combien de chrétiens protestants étaient du même avis que Monseigneur Pierre Marini Bodo dans sa confirmation de la véracité des résultats de l'élection présidentielle du 28 novembre 2011.

Avec le recul du temps, tout le monde reconnait la fausseté de résultats publiés par la CENI le 9 décembre 2011. Certains membres du Bureau de la CENI de l'époque commencent à délier leurs langues pour dire dans quelles conditions ces résultats moins vrais ont été publiés. La conséquence de ce tripatouillage électoral est l'illégitimité du pouvoir du Président Joseph Kabila qui a eu recours, deux ans après le début de son nouveau mandate, à des concertations nationales ayant abouti à la mise en place d'un Gouvernement de « cohésion nationale » pour gouverner. Peut-on recourir à cette pratique après avoir honnêtement gagné?

Monseigneur Pierre Marini Bodo est un Sénateur au mandat inconstitutionnel. Elu en 2007, le Sénat actuel devait terminer son mandat en février 2012. Dans le contexte de l'absence d'organisation des élections provinciales, la nouvelle Assemblée nationale cohabite avec un ancien Sénat où siège le Représentant légal de l'Eglise du Christ au Congo. Paradoxalement, il n'a jamais usé de son courage soit pour dénoncer la situation actuelle du Sénat congolais qui est une tricherie par rapport à la volonté du peuple.

Par contre, les musulmans se sont montrés réactifs. L'Imam Abdallah Mangala Luaba qui représentait la Communauté islamique en RDC a été contraint de quitter ses fonctions par ses pairs. Après avoir constaté que ce dernier avait dépassé son mandat et la volonté de quitter le poste commençait à manquer dans son chef, ses pairs l'ont contraint à partir. Il est parti difficilement après avoir gouté aux délices que lui procurait son statut de Représentant de la Communauté islamique. On l'a vu dans toutes les rencontres importantes même à Kampala (Ouganda) à l'occasion de négociations entre le Gouvernement et le M23. Si les musulmans ont réussi à rappeler le respect de la limite des mandats à leur Représentant, il n'en est pas le cas pour les Eglises du réveil. 
La présence des Eglises du Réveil dans cette plate-forme des Confessions religieuses pose la question cruciale de savoir si ces Eglises peuvent constituer une confession. La réponse à cette question est de la compétence des théologiens. Ce qui importe ici est de noter que le Représentant des Eglises du Réveil au sein des Confessions religieuses n'est pas reconnu par d'autres Eglises dont les chefs préfèrent entrer en contact direct avec les pouvoirs publics sans passer le Chef de leur « Confession ». Cet état de division et de guerre de leadership ne fait que profiter aux dirigeants politiques qui s'adonnent à la manipulation à sa guise.

\section{Conclusion}

L'un des problèmes à résoudre dans la voie de sortie de la crise congolaise est l'amélioration de la qualité de l'élite dirigeante dans toutes les institutions jusqu'au sein des communautés religieuses. La qualité d'hommes et de femmes qui nous dirigent est encore «médiocre ». La réaction de Monseigneur Pierre Marini Bodo à la déclaration du Cardinal Laurent Monsengwo est un des exemples de cette médiocrité de nos dirigeants. Il faut faire le ménage même au sein des Confessions religieuses. En l'état actuel des Confessions religieuses, elles ne joueront aucun rôle positif pour les élections de 2016.

Le premier signe visible de l'inféodation de certaines Confessions religieuses au pouvoir politique est l'élection de l'Abbé Appolinaire Malu-Malu pour diriger la CENI alors que cette personnalité ne donne pas le gage de son indépendance à cause de ses «affinités » avec le Président de la République. Son écrasante élection (7voix sur 8) par les représentants des Confessions religieuses n'a pas été obtenue sur la base des critères objectifs comme on a eu l'habitude de le faire croire. Sa récusation par une partie de l'Opposition politique et de l'Eglise catholique pose les germes d'une contestation des elections projetées en 2016. Cette responsabilité d'un éventuel blocage du processus électoral à cause de la personne d'Appollinaire Malumalu incombera aux Confessions religieuses. Le sort de l'association des Confessions religieuses est dans sa dissolution; car elle s'est transformée finalement en " confusion religieuse ». Elle devait disparaître pour que chaque Eglise ou communauté religieuse assume ses responsabilités devant Dieu, la nation et ses fidèles. 